\title{
On Dual $k$ - Pell Bicomplex Numbers and Some Identities Including Them
}

\author{
Serpil Halıcı $^{1 *}$ and Şule Çürük ${ }^{1}$ \\ ${ }^{1}$ Department of Mathematics, Faculty of Science and Arts, Pamukkale University, Denizli, Turkey \\ *Corresponding author : shalici@pau.edu.tr
}

\section{Article Info}

Keywords: Bicomplex numbers, Dual numbers, Pell numbers

2010 AMS: 11Y55, 11B39, $11 B 37$

Received: 11 April 2020

Accepted: 9 June 2020

Available online: 10 June 2020

\begin{abstract}
In the paper, we have considered the real and dual bicomplex numbers separately. Firstly, we examine the dual numbers and investigate the characteristic properties of them. Then, we give the definition, feature and related concepts about bicomplex numbers. And we define the number of dual $k$ - Pell bicomplex numbers that are not found for the first time in the literature and we examine the norm and conjugate properties of these numbers. We give equations about conjugates and give also some important characteristic of these newly defined numbers, and we write the recursive correlations of these numbers. Using these relations we give some important identities such as Vajda's, Honsberger's and d'Ocagne identities.
\end{abstract}

\section{Introduction}

The sequence $k-$ Pell is defined as follows[1]:

$$
\left\{P_{k, n}\right\}_{n \geq 0}=\left\{0,1,2,4+k, 8+4 k, 16+12 k+k^{2}, \ldots, 2 P_{k, n-1}+k P_{k, n-2}, \ldots\right\} .
$$

The elements of this set are satisfied the following relation:

$$
P_{k, n}=2 P_{k, n-1}+k P_{k, n-2}, k \in Z^{+}, n \geq 2
$$

and the initial values are

$$
P_{k, 0}=0, P_{k, 1}=1 .
$$

In [2], Binet-like formula related to these numbers is given as

$$
P_{k, n}=\frac{(1+\sqrt{1+k})^{n}-(1-\sqrt{1+k})^{n}}{2 \sqrt{1+k}} .
$$

The characteristic equation that gives these numbers and the roots of this equation are as follows:

$$
x^{2}-2 x-k=0, \alpha+\beta=2, \alpha \beta=-k, \alpha-\beta=2 \sqrt{1+k} .
$$


For more details on Pell sequence can be seen the references [3]. Bicomplex numbers is a 4- dimensional real vector space and it is defined as follows.

$$
B C=\left\{b=b_{1}+b_{2} i+b_{3} j+b_{4} i j: b_{1}, b_{2}, b_{3}, b_{4} \in R\right\} .
$$

Hence, any bicomplex number $b$ consists of a real and three imaginary units. It should be noted that the multiplication of bicomplex numbers is similar to multiplication of real quaternions. That is,

$$
i^{2}=j^{2}=-1, i j^{2}=1, i j=j i=k .
$$

It is noted that there are some differences between these two sets of numbers. According to this, we can list these as bicomplex numbers are commutative and they have zero divisors and non-trivial idempotent elements. On the other hand the real quaternions are non-commutative and don't have zero divisors and non-trivial idempotent elements. Also, the commutative property is satisfied for elements of the set $B C$.

In this work, we first investigate some properties of bicomplex numbers by examining the conjugates and norms. Then, we have introduced a new set of bicomplex numbers with coefficient from Pell number sequence, and gave some fundamental properties of this new set. Also, we gave some generalized identities such as Catalan's identity, d'Ocagne's identity, Honsberger formula, that the elements of this set provided. Working the mathematical structure of quantum mechanics on the bicomplex number field, there are many studies in this topic(see, [4]-[10]). $n-t h, k-$ Pell bicomplex number $B P_{k, n}$ is as follows:

$$
B P_{k, n}=P_{k, n}+i P_{k, n+1}+j P_{k, n+2}+i j P_{k, n+3} .
$$

That is the $k-$ Pell bicomplex number sequence is

$$
\left\{B P_{k, n}\right\}_{n \geq 0}=B P_{k, n}: B P_{k, n}=2 B P_{k, n-1}+k B P_{k, n-2}, k \in Z^{+}, n \geq 2 .
$$

Here $B P_{k, 0}=i+2 j+(4+k) i j$ and $B P_{k, 1}=1+2 i+(4+k) j+(8+4 k) i j$.

Follows from that we have

$$
B P_{k, 2}=2 B P_{k, 1}+k B P_{k, 0}=2+(4+k) i+(8+4 k) j+\left(16+12 k+k^{2}\right) i j .
$$

So, we can write

$$
B P_{k, n+1}=2 B P_{B} P_{k, n}+k B P_{B} P_{k, n-1}
$$

which is a useful equation.

\section{Dual $k$ - Pell bicomplex numbers}

As known that the dual numbers are binary members or a member of the 2 parameter families of the complex numbers system, called generalized complex numbers. Then, any dual number can be written as $z=x+\varepsilon y$, where $(x, y) \in R^{2}$ and $\varepsilon$ is a nilpotent number, also $\varepsilon^{2}=0$ and $\varepsilon \neq 0$. Then, the dual numbers set is

$$
D=R[\varepsilon]=\left\{z=x+\varepsilon y:(x, y) \in R^{2}, \varepsilon^{2}=0, \varepsilon \neq 0\right\} .
$$

Now, for the numbers $k \in Z^{+}$, we define dual $k-$ Pell number as follows:

$$
\widehat{P_{k, n}}=P_{k, n}+\varepsilon P_{k, n+1} \text {. }
$$

Hence, we can define any element of the dual bicomplex sequence $\widehat{\left\{B P_{k, n}\right\}_{n \geq 0}}$ as

$$
\widehat{B P_{k, n}}=B P_{k, n}+\varepsilon B P_{k, n+1} \text {. }
$$

Here $B P_{k, n}$ is the $n-t h, k-$ Pell bicomplex number.

Theorem 2.1. The elements of dual bicomplex sequence $\widehat{\left\{B_{k, n}\right\}_{n \geq 0}}$ are satisfied the following relation:

Where the initial values $\widehat{B P_{k, 0}}$ and $\widehat{B P_{k, 1}}$ are follows.

$$
\widehat{B P_{k, n}}=2 \widehat{B P_{k, n-1}}+k \widehat{B P_{k, n-2}}, n \geq 1 .
$$

$$
\widehat{B P_{k, 0}}=B P_{k, 0}+\varepsilon B P_{k, 1} \text { and } \widehat{B P_{k, 1}}=B P_{k, 1}+\varepsilon B P_{k, 2}
$$

respectively. 
Proof. For $n=2$, we have

$$
\widehat{B P_{k, 2}}=2 \widehat{B P_{k, 1}}+k \widehat{B P_{k, 0}}
$$

From the following the fact we get

$$
\widehat{B P_{k, 1}}=B P_{k, 1}+\varepsilon B P_{k, 2} \text { and } k \widehat{B P_{k, 0}}=k B P_{k, 0}+k \varepsilon B P_{k, 1} .
$$

So, we write

$$
2 \widehat{B P_{k, 1}}+k \widehat{B P_{k, 0}}=\left(2 B P_{k, 1}+k B P_{k, 0}\right)+\varepsilon\left(2 B P_{k, 2}+k B P_{k, 1}\right) .
$$

Follows from that, we have this:

$$
2\left(B P_{k, 1}+\varepsilon B P_{k, 2}\right)+k\left(B P_{k, 0}+\varepsilon B P_{k, 1}\right)=2 \widehat{B P_{k, 1}}+k \widehat{B P_{k, 0}}=\widehat{B P_{k, 2}} .
$$

In here the initial values are

$$
\widehat{B P_{k, 0}}=(i+2 j+(4+k) i j)+\varepsilon(1+2 i+(4+k) j+(8+4 k) i j)
$$

and

$$
\widehat{B P_{k, 1}}=1+2 i+(4+k) j+(8+4 k) i j+\varepsilon\left(2+(4+k) i+(8+4 k) j+\left(16+12 k+k^{2}\right) i j\right) .
$$

Furthermore, we can also write the number $\widehat{B P_{k, n}}$ differently as follows:

$$
\widehat{B P_{k, n}}=\left(P_{k, n}+i P_{k, n+1}+j P_{k, n+2}+i j P_{k, n+3}\right)+\varepsilon\left(P_{k, n+1}+i P_{k, n+2}+j P_{k, n+3}+i j P_{k, n+4}\right) .
$$

Then, we get

$$
\widehat{B P_{k, n}}=\widehat{P_{k, n}}+i \widehat{P_{k, n+1}}+j \widehat{P_{k, n+2}}+i j \widehat{P_{k, n+3}}
$$

where $\widehat{P_{k, n}}$ is the $n-t h$ dual $k-$ Pell number.

Since, usually the absolute values and arguments of bicomplex numbers are defined for each conjugation it is important to consider the conjugates of these numbers. Since there are four different units in this set, it means that four separate conjugates will be defined. According to this, for the bicomplex number $\widehat{B P_{k, n}}$, we can define four different conjugates as follows:

$$
\begin{aligned}
& \widehat{B P_{k, n}}=\widehat{P_{k, n}}-i \widehat{P_{k, n+1}}-j \widehat{P_{k, n+2}}-i j \widehat{P_{k, n+3}}, \\
& {\widehat{B P_{k, n}}}^{i}=\widehat{P_{k, n}}-i \widehat{P_{k, n+1}}+j \widehat{P_{k, n+2}}-i j \widehat{P_{k, n+3}}, \\
& {\widehat{B P_{k, n}}}^{j}=\widehat{P_{k, n}}+i \widehat{P_{k, n+1}}-j \widehat{P_{k, n+2}}-i j \widehat{P_{k, n+3}}, \\
& {\widehat{B P_{k, n}}}^{i j}=\widehat{P_{k, n}}-i \widehat{P_{k, n+1}}-j \widehat{P_{k, n+2}}+i j \widehat{P_{k, n+3}}
\end{aligned}
$$

Using this definition, we can give equations provided by conjugates. So, the following theorem is about them.

Theorem 2.2. For the numbers $\widehat{B P_{k, n}}$ the following equalities are satisfied:

$$
\begin{aligned}
& \widehat{B P_{k, n}}+\widehat{\overline{B P_{k, n}}}=2 \widehat{P_{k, n}} . \\
& \widehat{B P_{k, n}}+{\widehat{B P_{k, n}}}^{i}=2\left(\widehat{P_{k, n}}+j \widehat{P_{k, n+2}}\right) \text {. } \\
& \widehat{B P_{k, n}}+{\widehat{B P_{k, n}}}^{j}=2\left(\widehat{P_{k, n}}+i \widehat{P_{k, n+1}}\right) \text {. } \\
& \widehat{B P_{k, n}}+{\widehat{B P_{k, n}}}^{i j}=2\left(\widehat{P_{k, n}}+i j \widehat{P_{k, n+3}}\right) \text {. } \\
& {\widehat{B P_{k, n}}}^{i}+{\widehat{B P_{k, n}}}^{j}=2\left(\widehat{P_{k, n}}-i j \widehat{P_{k, n+3}}\right) \text {. } \\
& {\widehat{B P_{k, n}}}^{i}+{\widehat{B P_{k, n}}}^{i j}=2\left(\widehat{P_{k, n}}-i \widehat{P_{k, n+1}}\right) \text {. } \\
& {\widehat{B P_{k, n}}}^{j}+{\widehat{B P_{k, n}}}^{i j}=2\left(\widehat{P_{k, n}}-j \widehat{P_{k, n+2}}\right) \text {. } \\
& \widehat{B P_{k, n}}+{\widehat{B P_{k, n}}}^{i}+{\widehat{B P_{k, n}}}^{j}+{\widehat{B P_{k, n}}}^{i j}=4 \widehat{P}_{k, n} \text {. } \\
& \widehat{B P_{k, n}}-{\widehat{B P_{k, n}}}^{i}=-2 j \widehat{P_{k, n+2}} \text {. } \\
& \widehat{\overline{B P_{k, n}}}-{\widehat{B P_{k, n}}}^{i j}=-2 k \widehat{P_{k, n+3}} \text {. } \\
& {\widehat{B P_{k, n}}}^{i}-{\widehat{B P_{k, n}}}^{j}=-2\left(i \widehat{P_{k, n+1}}-j \widehat{P_{k, n+2}}\right) \text {. } \\
& {\widehat{B P_{k, n}}}^{i}-{\widehat{B P_{k, n}}}^{i j}=2 j\left(\widehat{P_{k, n+2}}-i \widehat{P_{k, n+3}}\right) \text {. }
\end{aligned}
$$


Proof. From the definition and properties of dual numbers the proofs can be easily seen.

Now, using the definitions norm and conjugate we also give the following theorem.

Theorem 2.3. For the numbers $\widehat{B P_{k, n}}$ the following equalities are satisfied:

$$
\begin{aligned}
& \text { i) } N r\left(\widehat{B P_{k, n}}\right)={\widehat{P_{k, n}}}^{2}+{\widehat{P_{k, n+1}}}^{2}+{\widehat{P_{k, n+2}}}^{2}-{\widehat{P_{k, n+3}}}^{2}+2\left(\widehat{i P_{k, n+2}} \widehat{P_{k, n+3}}+j \widehat{P_{k, n+1}} \widehat{P_{k, n+3}}-i j \widehat{P_{k, n+1}} \widehat{P_{k, n+2}}\right) \\
& \text { ii) } N r\left({\widehat{B P_{k, n}}}^{i}={\widehat{P_{k, n}}}^{2}+{\widehat{P_{k, n+1}}}^{2}-{\widehat{P_{k, n+2}}}^{2}-{\widehat{P_{k, n+3}}}^{2}+2 j\left(\widehat{P_{k, n} \widehat{P}_{k, n+2}}-\widehat{P_{k, n+1}} \widehat{P_{k, n+3}}\right)\right. \\
& \text { iii) } N r\left({\widehat{B P_{k, n}}}^{j}={\widehat{P_{k, n}}}^{2}-{\widehat{P_{k, n+1}}}^{2}+{\widehat{P_{k, n+2}}}^{2}-{\widehat{P_{k, n+3}}}^{2}+2 i\left(\widehat{\left(P_{k, n} \widehat{P}_{k, n+1}\right.}-\widehat{P_{k, n+2}} \widehat{P_{k, n+3}}\right)\right. \\
& \text { vi) } N r\left(\widehat{B P_{k, n}}\right)^{i j}={\widehat{P_{k, n}}}^{2}+{\widehat{P_{k, n+1}}}^{2}+{\widehat{P_{k, n+2}}}^{2}-{\widehat{P_{k, n+3}}}^{2}+2 i j\left(\widehat{P_{k, n} \widehat{P}_{k, n+3}}-\widehat{P_{k, n+1}} \widehat{P_{k, n+2}}\right)
\end{aligned}
$$

Proof. As per the definition of norm, we write

$$
\text { i) } \operatorname{Nr}\left(\widehat{B P_{k, n}}\right)=\left(\widehat{B P_{k, n}}\right)\left(\widehat{\overline{B P_{k, n}}}\right)=\left(\widehat{P_{k, n}}+i \widehat{P_{k, n+1}}+j \widehat{P_{k, n+2}}+i j \widehat{P_{k, n+3}}\right)\left(\widehat{P_{k, n}}-i \widehat{P_{k, n+1}}-j \widehat{P_{k, n+2}}-i j \widehat{P_{k, n+3}}\right) \text {. }
$$

After some calculations, we get

$$
N r\left(\widehat{B P_{k, n}}\right)={\widehat{P_{k, n}}}^{2}+{\widehat{P_{k, n+1}}}^{2}+{\widehat{P_{k, n+2}}}^{2}-{\widehat{P_{k, n+3}}}^{2}+2\left(i \widehat{P_{k, n+2} \widehat{P}_{k, n+3}}+j \widehat{P_{k, n+1}} \widehat{P_{k, n+3}}-i j \widehat{P_{k, n+1} \widehat{P}_{k, n+2}}\right) .
$$

In other cases, proof can be made in a similar way.

Note here that the dual $k$-Pell bicomplex numbers with the negative indices can be given.

Corollary 2.4. Negative dual $k$ - Pell bicomplex numbers $\widehat{B P_{k,-n}}$ are given as

$$
(-1)^{n-1}\left\{P_{k, n}-i P_{k, n-1}+j P_{k, n-2}-i j P_{k, n-3}+\varepsilon\left(-P_{k, n-1}+i P_{k, n-2}-j P_{k, n-3}+i j P_{k, n-4}\right)\right\} .
$$

Proof. From the equalities $P_{-n}=(-1)^{n-1} P_{n}$ and $P_{0}=0$, we get

$$
P_{k,-n}=(-1)^{n-1} P_{k, n}
$$

and writing negative of its instead of $n$ in the equation

$$
\begin{gathered}
B P_{k, n}=P_{k, n}+i P_{k, n+1}+j P_{k, n+2}+i j P_{k, n+3}, \\
B P_{k,-n}=(-1)^{n-1} P_{k, n}+i(-1)^{n} P_{k, n-1}+j(-1)^{n+1} P_{k, n-2}+i j(-1)^{n+2} P_{k, n-3}
\end{gathered}
$$

can be written. It follows from that

$$
B P_{k,-n}=(-1)^{n-1}\left(P_{k, n}-i P_{k, n-1}+j P_{k, n-2}-i j P_{k, n-3}\right) .
$$

On the other hand for dual of these numbers, by the aid of the equality

$$
\widehat{B P_{k, n}}=B P_{k, n}+\varepsilon B P_{k, n+1}
$$

we have

$$
\widehat{B P_{k,-n}}=B P_{k,-n}+\varepsilon B P_{k,-n+1} .
$$

Hence, the term $\widehat{B P_{k,-n}}$ is as follows:

$$
(-1)^{n-1}\left(P_{k, n}-i P_{k, n-1}+j P_{k, n-2}-i j P_{k, n-3}\right)+\varepsilon(-1)^{n}\left(P_{k, n-1}-i P_{k, n-2}+j P_{k, n-3}-i j P_{k, n-4}\right) .
$$

That is we have

$$
\widehat{B P_{k,-n}}=(-1)^{n-1}\left\{P_{k, n}-i P_{k, n-1}+j P_{k, n-2}-i j P_{k, n-3}+\varepsilon\left(-P_{k, n-1}+i P_{k, n-2}-j P_{k, n-3}+i j P_{k, n-4}\right)\right\}
$$

which is desired result. 
Generating functions and their properties are a powerful tool for solving recurrences and combinatorial problems. Generally, a generating function is a series of formal power containing the information of the inputs of a given sequence in its coefficients. There are various generating functions according to usage and application areas.

In the following theorem, the generating function will be given for the dual $k$ - Pell bicomplex sequence.

Theorem 2.5. The function that generates the elements of the sequence $\left\{\widehat{B P_{k, n}}\right\}_{n \geq 0}$ is

$$
G(t)=\frac{\widehat{B P_{k, 0}}+\left(\widehat{B P_{k, 1}}-2 \widehat{B P_{k, 0}}\right)}{1-2 t-k t^{2}}
$$

Here $\widehat{B P_{k, 0}}$ and $\widehat{B P_{k, 1}}$ are

$$
i+2 j+(4+k) i j+(1+2 i+(4+k) j+(8+4 k) i j)
$$

and

$$
1+2 i+(4+k) j+(8+4 k) i j+\varepsilon\left(2+(4+k) i+(8+4 k) j+\left(16+12 k+k^{2}\right) i j\right) .
$$

Proof. The generating function of $\left\{\widehat{B P_{k, n}}\right\}_{n \geq 0}$ is as follows:

$$
\begin{aligned}
& g_{\widehat{B P_{k, n}}} t==G(t)=\sum_{n=0}^{\infty} \widehat{B P_{k, n}} t^{n}, \\
& G(t)=\widehat{B P_{k, 0}}+\widehat{B P_{k, 1}} t+\widehat{B P_{k, 2}} t^{2}+\ldots+\widehat{B P_{k, n}} t^{n}+\ldots, \\
& -2 t G(t)=-2\left(\widehat{B P_{k, 0}} t+\widehat{B P_{k, 1}} t^{2}+\widehat{B P_{k, 2}} t^{3}+\ldots+\widehat{B P_{k, n}} t^{n}+\widehat{B P_{k, n+1}} t^{n+1} \ldots\right), \\
& -k t^{2} G(t)=-k\left(\widehat{B P_{k, 0}} t^{2}+\widehat{B P_{k, 1}} t^{3}+\widehat{B P_{k, 2}} t^{4}+\ldots+\widehat{B P_{k, n}} t^{n+1}+\widehat{B P_{k, n}} t^{n+2} \ldots\right) .
\end{aligned}
$$

Using above equations, we write the following formula:

$$
\left(1-2 t-k t^{2}\right) G(t)=\widehat{B P_{k, 0}}+\left(\widehat{B P_{k, 1}}-2 \widehat{B P_{k, 0}}\right) t .
$$

Then, it follows that

$$
G(t)=\frac{\widehat{B P_{k, 0}}+\left(\widehat{B P_{k, 1}}-2 \widehat{B P_{k, 0}}\right) t}{1-2 t-k t^{2}}
$$

that the desired generating function. Here $\widehat{B P_{k, 0}}$ and $\widehat{B P_{k, 1}}$ are

$$
\widehat{B P_{k, 0}}=i+2 j+(4+k) i j+\varepsilon(1+2 i+(4+k) j+(8+4 k) i j)
$$

and

$$
\widehat{B P_{k, 1}}=1+2 i+(4+k) j+(8+4 k) i j+\varepsilon\left(2+(4+k) i+(8+4 k) j+\left(16+12 k+k^{2}\right) i j\right)
$$

respectively.

Theorem 2.6. Elements of the sequence $\left\{\widehat{B P_{k, n}}\right\}_{n \geq 0}$ satisfy in the following formula:

$$
\widehat{B P_{k, n}}=\frac{\underline{\alpha} \alpha^{n}-\underline{\beta} \beta^{n}}{\alpha-\beta}
$$

where

$$
\underline{\alpha}=1+i \alpha+j \alpha^{2}+i j \alpha^{3}+\varepsilon\left(\alpha+i \alpha^{2}+j \alpha^{3}+i j \alpha^{4}\right)
$$

and

$$
\underline{\beta}=-\left\{\left(1+i \beta+j \beta^{2}+i j \beta^{3}\right)+\varepsilon\left(\beta+i \beta^{2}+j \beta^{3}+i j \beta^{4}\right)\right\}
$$

Proof. The general solution of the characteristic equation of the sequence $\left\{\widehat{B P_{k, n}}\right\}_{n \geq 0}$ is

$$
\widehat{B P_{k, n}}=A \alpha^{n}+B \beta^{n}
$$


The initial conditions $\widehat{B P_{k, 0}}$ and $\widehat{B P_{k, 1}}$ yields the following equations

$$
\widehat{B P_{k, 0}}=A+B
$$

and

$$
\widehat{B P_{k, 1}}=A \alpha+B \beta
$$

respectively. Solving these equations, we get

$$
A=\frac{\widehat{B P_{k, 1}}-\beta \widehat{B P_{k, 0}}}{\alpha-\beta}, B=\frac{\alpha \widehat{B P_{k, 0}}-\widehat{B P_{k, 1}}}{\alpha-\beta} .
$$

So, we have the following formula for the sequence $\left\{\widehat{B P_{k, n}}\right\}_{n \geq 0}$ :

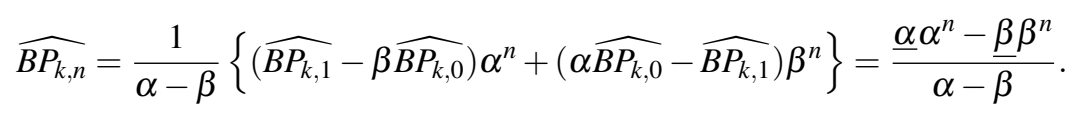

We also note that the remarkable fact the last formula can be rewritten as follows:

$$
\widehat{B P_{k, n}}=\widehat{B P_{k, 1}} P_{k, n}+k \widehat{B P_{k, 0}} P_{k, n-1}
$$

or

$$
\widehat{B P_{k, n}}=B P_{k, 1} \widehat{P_{k, n}}+k B P_{k, 0} \widehat{P_{k, n-1}}
$$

Here, the values $P_{k, n}, P_{k, n-1}, B P_{k, 1}$ and $B P_{k, 0}$ are known.

The relation given in the theorem above theorem is known as the Binet formula. Many identities related to all Fibonacci-like integer sequences are obtained with the help of this formula.

Theorem 2.7. The Cassini's identity for the sequence $\left\{\widehat{B P_{k, n}}\right\}_{n \geq 0}$ is follows:

$$
\widehat{B P_{k, n-1}} \widehat{B P_{k, n+1}}-{\widehat{B P_{k, n}}}^{2}=(-1)^{n} \underline{\alpha} \underline{\beta}^{n-1}
$$

where $\underline{\alpha} \beta$ is equal to this:

$$
(1+k)\left\{k^{2}-1-2 i(1-k)-2 j(k+2)+8 i j\right\}-2 \varepsilon\left\{(1+k)-k^{2}(1+k)+2 i\left(1-k^{2}\right)+2 j\left(k^{2}+3 k+2\right)+4 i j(2+k)\right\} .
$$

Proof. If we use the Binet formula for proof, then we get

$$
\begin{gathered}
\widehat{B P_{k, n-1}} \widehat{B P_{k, n+1}}-{\widehat{B P_{k, n}}}^{2}=\frac{1}{4(1+k)}\left\{\left(\underline{\alpha} \alpha^{n-1}-\underline{\beta} \beta^{n-1}\right)\left(\underline{\alpha} \alpha^{n+1}-\underline{\beta} \beta^{n+1}\right)-\left(\underline{\alpha} \alpha^{n}-\underline{\beta} \beta^{n}\right)^{2}\right\} . \\
\widehat{B P_{k, n-1}} \widehat{B P_{k, n+1}}-{\widehat{B P_{k, n}}}^{2}=\frac{1}{4(1+k)}\left(\underline{\alpha}^{2} \alpha^{2 n}-\underline{\alpha} \underline{\beta} \alpha^{n-1} \beta^{n+1}-\underline{\alpha} \underline{\beta} \beta^{n-1} \alpha^{n+1}+\underline{\beta}^{2} \beta^{2 n}-\underline{\alpha}^{2} \alpha^{2 n}+2 \underline{\alpha} \underline{\beta} \alpha^{n} \beta^{n}-\underline{\beta^{2}} \beta^{2 n}\right) .
\end{gathered}
$$

If the required simplifications are made, then

$$
\widehat{B P_{k, n-1}} \widehat{B P_{k, n+1}}-{\widehat{B P_{k, n}}}^{2}=(-1)^{n} \underline{\alpha} \underline{\beta} k^{n-1}
$$

is obtained. Thus, the proof is completed.

Theorem 2.8. The Catalan's identity for the sequence $\left\{\widehat{B P_{k, n}}\right\}_{n \geq 0}$ is

$$
\widehat{B P_{k, n+m}} \widehat{B P_{k, n-m}}-{\widehat{B P_{k, n}}}^{2}=\frac{(-1)^{n-m+1} k^{n-m}}{4(1-k)} \underline{\alpha} \underline{\beta}\left\{\alpha^{2 m}+\beta^{2 m}-2(-k)^{m}\right\} .
$$


Proof. From the Binet formula, we get the following equation.

$$
\widehat{B P_{k, n+m}} \widehat{B P_{k, n-m}}-{\widehat{B P_{k, n}}}^{2}=\frac{1}{4(1+k)}\left\{\left(\underline{\alpha} \alpha^{n+m}-\underline{\beta} \beta^{n+m}\right)\left(\underline{\alpha} \alpha^{n-m}-\underline{\beta} \beta^{n-m}\right)-\left(\underline{\alpha} \alpha^{n}-\underline{\beta} \beta^{n}\right)^{2}\right\} .
$$

If necessary corrections are made,

$$
\widehat{B P_{k, n+m}} \widehat{B P_{k, n-m}}-{\widehat{B P_{k, n}}}^{2}=\frac{(-1)^{n-m+1} k^{n-m}}{4(1-k)} \underline{\alpha}\left\{\alpha^{2 m}+\beta^{2 m}-2(-k)^{m}\right\} .
$$

is obtained. Thus, the proof is completed.

Specially, in the Catalan identity, if we take $m=1$ then we get the Cassini's identity.

Theorem 2.9. The Honsberger's identity for the sequence $\left\{\widehat{B P_{k, n}}\right\}_{n \geq 0}$ is

$\left.\widehat{B P_{k, m-1}} \widehat{B P_{k, n}}+\widehat{B P_{k, m}} \widehat{B P_{k, n+1}}=\frac{1}{4(1+k)}\left\{\underline{\alpha}^{2} \alpha^{n+m-1}\left(1+\alpha^{2}\right)+\underline{\beta}^{2} \beta^{n+m-1}\left(1+\beta^{2}\right)-\underline{\alpha} \underline{\beta}(-k)^{m-1} \alpha^{n-m+1}+\beta^{n-m+1}\right)(1-k)\right\}$.

Here,

$\underline{\alpha}^{2}=\left(1-\alpha^{2}\right)\left(1-\alpha^{4}\right)+2 i \alpha\left(1-\alpha^{4}\right)+2 j \alpha^{2}\left(1-\alpha^{2}\right)+4 i j \alpha^{3}+2 \alpha \varepsilon\left\{\left(1-\alpha^{2}\right)\left(1-\alpha^{4}\right)+2 i \alpha\left(1-\alpha^{4}\right)+2 j \alpha^{2}\left(1-\alpha^{2}\right)+4 i j \alpha^{3}\right\}$.

$\underline{\beta}^{2}=\left(1-\beta^{2}\right)\left(1-\beta^{4}\right)+2 i \beta\left(1-\beta^{4}\right)+2 j \beta^{2}\left(1-\beta^{2}\right)+4 i j \beta^{3}+2 \beta \varepsilon\left\{\left(1-\beta^{2}\right)\left(1-\beta^{4}\right)+2 i \beta\left(1-\beta^{4}\right)+2 j \beta^{2}\left(1-\beta^{2}\right)+4 i j \beta^{3}\right\}$.

Proof. Let us use the Binet formula. Then

$$
\begin{aligned}
& \widehat{B P_{k, m-1}} \widehat{B P_{k, n}}+\widehat{B P_{k, m}} \widehat{B P_{k, n+1}}=\frac{1}{4(1+k)}\left\{\left(\underline{\alpha} \alpha^{m-1}-\underline{\beta} \beta^{m-1}\right)\left(\underline{\alpha} \alpha^{n}-\underline{\beta} \beta^{n}\right)+\left(\underline{\alpha} \alpha^{m}-\underline{\beta} \beta^{m}\right)\left(\underline{\alpha} \alpha^{n+1}-\underline{\beta} \beta^{n+1}\right)\right\} . \\
= & \frac{1}{4(1+k)}\left(\underline{\alpha}^{2} \alpha^{n+m-1}-\underline{\alpha} \underline{\beta} \alpha^{m-1} \beta^{n}-\underline{\alpha} \underline{\beta} \alpha^{n} \beta^{m-1}+\underline{\beta}^{2} \beta^{n+m-1}+\underline{\alpha}^{2} \alpha^{n+m+1}-\underline{\alpha} \underline{\beta} \alpha^{m} \beta^{n+1}-\underline{\alpha} \underline{\beta} \beta^{m} \alpha^{n+1}+\underline{\beta}^{2} \beta^{n+m+1}\right)
\end{aligned}
$$

can be written. When the necessary actions are performed, we get the following equation.

$$
=\frac{1}{4(1+k)}\left\{\left(\underline{\alpha}^{2} \alpha^{n+m-1}\left(1+\alpha^{2}\right)+\underline{\beta}^{2} \beta^{n+m-1}\left(1+\beta^{2}\right)-\underline{\alpha} \underline{\beta}(-k)^{m-1}\left(\alpha^{n-m+1}+\beta^{n-m+1}\right)(1-k)\right\} .\right.
$$

Thus, the proof is completed.

Theorem 2.10. The d'Ocagne identity for the sequence $\left\{\widehat{B P_{k, n}}\right\}_{n \geq 0}$ is

$$
\widehat{B P_{k, m}} \widehat{B P_{k, n+1}}-\widehat{B P_{k, n}} \widehat{B P_{k, m+1}}=\frac{-\underline{\alpha}}{4(1+k)}\left\{(-k)^{m}\left(\beta^{n-m+1}+\alpha^{n-m+1}\right)-(-k)^{n}\left(\beta^{m-n+1}+\alpha^{m-n+1}\right)\right\} .
$$

Proof. Binet formula can be used to prove the proof. So,

$$
\widehat{B P_{k, m}} \widehat{B P_{k, n+1}}-\widehat{B P_{k, n}} \widehat{B P_{k, m+1}}
$$

is equal to this:

$$
\frac{1}{4(1+k)}\left\{\left(\underline{\alpha} \alpha^{m}-\underline{\beta} \beta^{m}\right)\left(\underline{\alpha} \alpha^{n+1}-\underline{\beta} \beta^{n+1}\right)-\left(\underline{\alpha} \alpha^{n}-\underline{\beta} \beta^{n}\right)\left(\underline{\alpha} \alpha^{m+1}-\underline{\beta} \beta^{m+1}\right)\right\} .
$$

When the necessary arrangements are performed, we get

$$
\widehat{B P_{k, m}} \widehat{B P_{k, n+1}}-\widehat{B P_{k, n}} \widehat{B P_{k, m+1}}=\frac{-\underline{\alpha}}{4(1+k)}\left\{\alpha^{m} \beta^{n+1}+\beta^{m} \alpha^{n+1}-\alpha^{n} \beta^{m+1}-\beta^{n} \alpha^{m+1}\right\}
$$

Thus, we get the desired result. That is,

$$
\widehat{B P_{k, m}} \widehat{B P_{k, n+1}}-\widehat{B P_{k, n}} \widehat{B P_{k, m+1}}=\frac{-\underline{\alpha} \underline{\beta}}{4(1+k)}\left\{(-k)^{m}\left(\beta^{n-m+1}+\alpha^{n-m+1}\right)-(-k)^{n}\left(\beta^{m-n+1}+\alpha^{m-n+1}\right)\right\} .
$$


Theorem 2.11. For the positive integers $n, i, j$, Vajda's identity related with the sequence $\left\{\widehat{B P_{k, n}}\right\}_{n \geq 0}$ is follows:

$$
\widehat{B P_{k, n+i}} \widehat{B P_{k, n+j}}-\widehat{B P_{k, n}} B \widehat{P_{k, n+i}+j}=\frac{(-1)^{n+1} k^{n}}{4(1+k)}\left\{\underline{\alpha} \underline{\beta}\left(\beta^{j}-\alpha^{j}\right)\left(\alpha^{i}-\beta^{i}\right)\right\} .
$$

Proof. Let us use the Binet formula for the proof. The desired this value, that is

$$
\widehat{B P_{k, n+i}} \widehat{B P_{k, n+j}}-\widehat{B P_{k, n}} B \widehat{P_{k, n+i}+j}
$$

is follows:

$$
\frac{1}{4(1+k)}\left\{\left(\alpha^{n+i}-\beta^{n+i}\right)\left(\alpha^{n+j}-\beta^{n+j}\right)-\left(\alpha^{n}-\beta^{n}\right)\left(\alpha^{n+i+j}-\beta^{n+i+j}\right)\right\} .
$$

When the necessary algebraic operations are performed, we get

$$
\frac{-\underline{\alpha} \underline{\beta}}{4(1+k)}\left\{\alpha^{n+i} \beta^{n+j}+\beta^{n+i} \alpha^{n+j}-\alpha^{n} \beta^{n+i+j}-\beta^{n} \alpha^{n+i+j}\right\} .
$$

From here, we get

$$
\widehat{B P_{k, n+i}} \widehat{B P_{k, n+j}}-\widehat{B P_{k, n}} B \widehat{P_{k, n+i+j}}=\frac{(-1)^{n+1} k^{n}}{4(1+k)}\left\{\underline{\alpha} \underline{\beta}\left(\beta^{j}-\alpha^{j}\right)\left(\alpha^{i}-\beta^{i}\right)\right\} .
$$

which is desired.

\section{Conclusion}

In this study, we examine the dual numbers and give them the characteristics of these numbers. Also, we give the definition and related concepts about bicomplex numbers. Moreover, we define the number of dual $k-$ Pell bicomplex numbers which are not found for the first time in the literature and we examined the norm and conjugate properties of these numbers. We have given some important characteristics of these newly defined numbers, and we have obtain the recursive relations of these numbers. Using obtained relations one can investigate the other important identities.

\section{Acknowledgement}

We would like to thank the referees who reviewed this work and submitted their positive reviews.

\section{References}

[1] P. Catarino, On some identities and generating functions for $k$-Pell numbers, Int. J. of Math. Anal., 7(38) (2013), $1877-1884$.

[2] P. Catarino, Bicomplex $k$ - Pell quaternions, Comput. Methods Funct. Theory, 19(1) (2019), 65-76.

[3] S. Halici, On Some Pell Polynomials. Acta Uni. Apul., 29(2012), 105-112.

[4] D. Alpay, M. E. Luna-Elizarrarás, M. Shapiro, D. C. Struppa, Basics of Functional Analysis with Bicomplex Scalars, and Bicomplex Schur analysis. Springer Sci. and Business Media,(2014)

[5] F. Babadag, Fibonacci, Lucas numbers with dual bicomplex numbers, J. Math. Sci., 10(1-2) (2018), 161-172.

[6] A. T. Benjamin, S. S. Plott, J. A. Sellers, Tiling proofs of recent sum identities involving Pell numbers, Ann. Comb., 12(3) (2008), $271-278$.

[7] M. A. Gungor, A. Cihan, On dual-hyperbolic numbers with generalized Fibonacci and Lucas numbers components, Fundam. J. Math. Appl., 2(2) (2019), 162-172, doi: 10.33401/fujma.617415.

[8] S. Halici, On Bicomplex Fibonacci Numbers and Their Generalization, In Models and Theories in Social Systems (pp. 509-524), Springer, Cham, (2019).

[9] S. Ö. Karakuş, F. K. Aksoyak, Generalized bicomplex numbers and Lie groups, Adv. Appl. Clifford Alg., 25(4) (2015), 943-963.

[10] M. E. Luna-Elizarraras, M. Shapiro, D. C. Struppa, A. Vajiac, Bicomplex numbers and their elementary functions, Cubo(Temuco), 14(2) (2012), 61-80. 